\title{
Phase diagrams of flux lattices with disorder
}

\author{
T. Giamarchi \\ Laboratoire de Physique des Solides, Univergité Paris-Sud, \\ Bât. 510, 91405 Orsay, France* \\ P. Le Doussal \\ CNRS-Laboratoire de Physique Théorique de l'Ecole \\ Normale Supérieure, 24 rue Lhomond, F-75231 Pari *** $^{2}$
}

(August 15, 2018)

\begin{abstract}
We review the prediction, made in a previous work [Phys. Rev. B 52 (1995)], that the phase diagram of type II superconductors consists of a topologically ordered Bragg glass phase at low fields undergoing a transition at higher fields into a vortex glass or a liquid. We estimate the position of the phase boundary using a Lindemann criterion. We find that the proposed phenomenology is compatible with recent experiments on superconductors.
\end{abstract}

It is remarkable that after a decade of experimental and theoretical efforts, the phase diagram of type II superconductors in a field is far from being completely elucidated [1]. Stimulated by the discovery of the high Tc materials, a reexamination of the mean-field phase diagram unravelled two main new phenomena. First, it was realized [2,3], and observed [4] that due to enhanced thermal fluctuations the Abrikosov lattice melts well below $H_{c 2}$ into a to a flux liquid. Secondly, it was argued that in the solid phase, pointlike disorder could produce a glassy state with barriers $U(j)$ diverging at small $j$, and thus characterized by the true vanishing of the linear resistivity even at finite temperature [5.,6]. This was a significant departure from traditional models of thermally assisted flux flow, which assumed finite barriers between pinned states. A precursor sign of an instability towards a glass was also found in the flux liquid [7]. Both for the technological applications of high- $T_{c}$ materials and from a purely theoretical point of vue, the understanding of the detailed properties of such a glassy phase is of paramount importance.

Two main phenomenological theories have been put forward to describe this glassy phase and to account for some of its properties observed in early experiments, mainly the observed continuous transition from the glass to the liquid and the giant thermal creep. The first approach is based on the gauge glass model [5,8], and assumes a complete destruction of the Abrikosov lattice. The second approach retains the the elastic lattice structure at small scale [6]. Although different in nature, both theories agreed that the disorder essential to produce the glassy low temperature phase and the vanishing of the linear resistivity, was also destroying at large scales the perfect flux lattice existing in mean field theory. The low temperature phase was therefore generally expected to be a topologically disordered phase, lacking translational order. Several calculations supported this point of view.
Elastic theory predicted at best a stretched exponential decay of translational order [6,9,10] (i.e. a power law grow of displacements) and general arguments tended to prove that disorder would always favor the presence of dislocations [8]. The vortex lattice seemed to be buried for good.

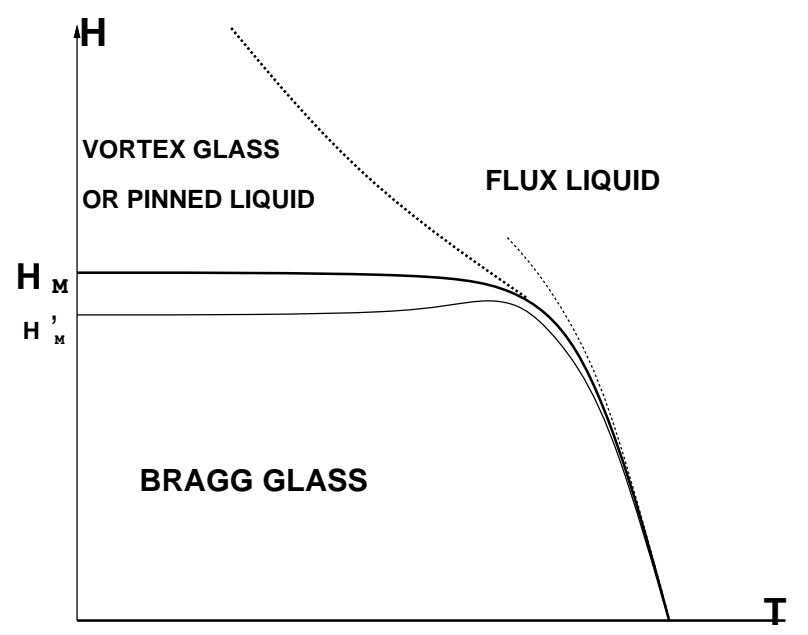

FIG. 1. The stability region of the Bragg glass phase in the magnetic field $H$, temperature $T$ plane is shown schematically (thick solid line). Upon increasing disorder the region shrinks as indicated by the thin solid line (see text). The melting line of the pure system is shown as a dotted line, and the vortex glass transition line (or crossover to the pinned liquid) is shown thick dotted.

A few points were not naturally fitting in the framework of these theories. Experimentally, a first order transition between the glass phase and the liquid was observed at low fields [11, 12] rather than the predicted continuous transition, observed at high fields. Also, decoration experiments of the flux lattice at very low fields 
$(60 \mathrm{G})$ in several materials were showing remarkably large regions free of dislocations 13. On the side of theory, old calculations on the related disordered elastic random field XY model 14 as well as more recent scaling arguments for the vortex lattice [15] suggested, within a purely elastic decription, a slower, logarithmic, growth of deformations.

In a recent work we have obtained the first quantitative theory of the elastic vortex lattice [16,17 in presence of point disorder. Contrarily to previous approaches, it provides a description valid at all scales and demonstrates that while disorder produces algebraic growth of displacements at short length scales, periodicity takes over at large scales and results in a decay of translational order at most algebraic 18]. One striking prediction is thus the existence of a glass phase with Bragg diffraction peaks ! This result was derived within an elastic theory, assuming the absence of dislocations. However, the very result of our calculation, i.e that quasi long range order survives, led us to advocate that dislocations would be much less relevant than commonly assumed [16]. The alleged importance of dislocations in a disordered system [8] made it mandatory to further investigate carefully whether dislocations would indeed modify the above result. The striking result [17] that we found based on energy arguments is that dislocations are not favorable for weak disorder in $d=3$. This implies self-consistently the existence of a thermodynamic glass phase, as far as energy and very low current transport properties are concerned, retaining a nearly perfect (i.e. algebraic) translational order. Since this phase exhibits Bragg peaks very much like a perfect lattice it was christened the "Bragg glass". Because it retains a "lattice" structure and Bragg peaks, this glass phase is radically different from the vortex glass picture based on a random gauge model. In particular, since such a phase is nearly as good as perfect lattice as far as translational order is concerned, it is natural to expect it to melt through a first order phase transition. We proposed [17] that the phase seen experimentally at low fields was in fact the Bragg glass, solving the apparent impossibility of a pinned solid. This allowed to account naturally for the first-order transition and the decoration experiments. Our prediction [17] that a new phase without topological defects, should be stable at weak disorder received subsequent further support both from numerical simulations 21, 22, and from analytical calculations in a layered geometry 23,24].

Once the existence of a weak disorder/low field Bragg glass phase is established the question arises of determining its limits of stability and phase boundaries. The Bragg glass phase should be stable as a self-consistent solution in the elastic limit, i.e as long as $R_{a} \gtrsim a$ [17]. This condition is violated when the field is increased and we proposed in 17] that upon raising the field the Bragg glass should undergo a transition into another phase, which could be a pinned liquid or another glass (vor- tex glass). A natural possibility then was that the critical point occuring on the melting line [12 was the end point of the transition line between the Bragg glass at low fields and a topologically disordered glassy phase (or a strongly pinned liquid) at higher field. We pointed out that the fact that this point can be lowered by adding impurities was a hint that it was related to this transition. Such a field-driven transition corresponds to the destruction of the Bragg glass by proliferation of topological defects upon raising the field, which is equivalent to increasing the effective disorder. The other transition from the thermal liquid into the putative superconducting state at higher fields is presumably continuous. The topology of the phase diagram proposed in [17] is as depicted in Fig. 1.

Several recent experiments can be interpreted to confirm the picture proposed in [17]. Neutron experiments can be naturally interpreted in term of the Bragg glass 26,27. In BSCCO neutron peaks are observed at low fields and disappear upon raising the field [28]. The phase diagram of BSCCO has been recently explored in details by overcoming spurious effects due to geometrical barriers 29]. It can increasingly be interpreted as a confirmation of our theory, the so-called second magnetization peak line being the candidate for the predicted field driven transition. Since our proposal this line has been investigated in more details in BSCCO [30] and found to be relatively temperature independent at lower temperatures and to be shifted downwards upon increase of point disorder through electron irradiation [31]. Also, similar types of phase diagrams are observed in a variety of materials, including YBCO 12,39. The fact that a controlled increase of point disorder through electron irradiation shifts the transition line to lower fields [25] is a strong indication that our picture is relevant in these materials as well.

In the present paper we follow on the theory exposed in [17. We make more quantitative estimates of the phase diagram depicted in Fig. 1 using a generalized Lindemann criterion. We also explore in more details some experimental consequences of our theory.

Let us consider a vortex lattice system in presence of disorder. We can model the vortex lattice by stacks of coupled planes. The system is therefore described by layers of two dimensional triangular lattices of vortices. We denote by $R_{i}$ the equilibrium position of the vortices in the absence of disorder, labeled by an integer $i$, in the $x y$ plane, and by $u\left(R_{i}, z\right)$ their in-plane displacements which are two dimensional vectors, (the vortex can only move within the plane). $z$ is the coordinate perpendicular to the planes and along the magnetic field and $x=(r, z)$. The total energy is:

$$
H=\frac{1}{2} \int d^{2} r d z\left[\left(c_{11}-c_{66}\right)\left(\partial_{\alpha} u_{\alpha}\right)^{2}+c_{66}\left(\partial_{\alpha} u_{\beta}\right)^{2}+\right.
$$




$$
\left.c_{44}\left(\partial_{z} u_{\alpha}\right)^{2}\right]+\int d^{2} r d z V(r, z) \rho(r, z)
$$

where the density of vortex lines is simply defined by $\rho(x)=\sum_{i} \delta^{(2)}\left(r-R_{i}-u\left(R_{i}, z\right)\right)$. The last term in (1) is the coupling to disorder. In the limit where many weak impurities act collectively on a vortex, point disorder can be modelled by a gaussian random potential $V(x)$ with correlations: $\overline{V(x) V\left(x^{\prime}\right)}=\Delta\left(r-r^{\prime}\right) \delta\left(z-z^{\prime}\right)$ where $\Delta(r)$ is a short range function [20] of range $\xi$ (the superconducting coherence length)

$$
\Delta(r)=d U_{p}^{2} e^{-r^{2} / \xi^{2}}
$$

where $d$ is the distance between layers and $U_{p}$ a typical pinning energy per unit length along $z$.

In the high- $T_{c}$ Abrikosov lattice, one has in principle to use non local elasticity, and the calculation along the lines of [17 can be done simply by using the complete known wavevector dependent expressions [3.32] of the elastic constants in (11). Since we are only interested in nearest neighbor correlations and want to obtain only an order of magnitude of the scales involved we use simple constant elastic moduli. The physical properties of (11) were examined in details in 16, 17] and we just recall here the results needed for the phase diagram. The mean squared relative displacements of two vortices separated by a distance $r$ is:

$$
B(r)=\overline{\left\langle(u(0,0)-u(r, 0))^{2}\right\rangle}
$$

where \langle\rangle denotes the thermal average, whereas $\ldots$ is the disorder average. From $B(r)$ one defines two length scales $R_{c}$ and $R_{a}$ in the $x y$ plane (and similarly $L_{c}$ and $L_{a}$ along $z$ ) such that $B_{\text {dis }}\left(R_{c}\right) \sim \max \left(\xi^{2},\left\langle u^{2}\right\rangle_{T}\right)$ (see below) and $B\left(R_{a}\right) \sim a^{2}$ respectively. $R_{c}$ is the Larkin-Ovchinikov pinning length [33] directly related to the critical current, whereas $R_{a}$ is the scale at which one enters the asymptotic regime with a logarithmic growth of the displacements. The model (11) leads to the Bragg glass phase with quasi long range translational order.

To determine the region of stability of the Bragg glass phase, we follow the arguments proposed in 17] that the elastic structure will become unstable when the displacement between two neighbors becomes of order of the lattice spacing $a$, i.e

$$
B(r=a) \sim a^{2}
$$

To be more quantitative, one can introduce, as for the normal thermal melting a Lindermann constant $c_{L}$ and take for the criterion of stability of the Bragg glass phase

$$
B(r=a)=\overline{\left\langle(u(0,0)-u(a, 0))^{2}\right\rangle}=c_{L}^{2} a^{2}
$$

$c_{L}$, the Lindemann constant is usually of the order of $c_{L} \sim 0.1-0.2$ in usual melting and we make here the assumption that $c_{L}$ is indeed a constant independent of the field.

From (5) one sees that both disorder and thermal fluctuations act together to increase the displacements. In fact the formula (3.18) of 116,17] shows that $B(r=a)$ splits naturally in two parts $B(r=a) \approx 2\left\langle u^{2}\right\rangle_{T}+$ $B_{d i s}(r=a)$. One immediate consequence of (5) is therefore that the melting line should be pushed downwards in the presence of point like disorder. In fact the Bragg glass can disappear in two ways: (i) if the temperature is raised, it will melt to a liquid phase (ii) if the field is raised, which amounts to vary the effective disorder in the system, the system can become so disordered even at short length scales that dislocations will appear. (5) gives thus the limit of stability of the BG phase in the $H-T$ plane. Although the complete "melting" curve can be computed using the formulas for $B(r)$ obtained in 16, 17, such a calculation is tedious and offers little insight. We therefore study mainly here the two limits of low temperature where the transition is mainly fielddriven, and for temperature close to the melting curve in the absence of disorder.

If the temperature is close to the pure melting line, $B(r=a)$ is dominated by the thermal fluctuations. Since for weak disorder $R_{a} \gg a$ the disorder induced displacements are negligeable at the scale of nearest neighbors and one can compute (5) using thermal fluctuations only. One then easily recovers the pure melting line

$$
T_{m} \approx 4 a^{3} \sqrt{c_{66} c_{44}} c_{L}^{2}
$$

Disorder effects will push the melting line slightly down, but effects should be negligible at low field for which the effective disorder is small enough. Upon increasing the field disorder induced displacements will increase, forcing the transition line defined by (5) to go down to zero temperature at a finite field $H_{M}$. The scale at which disorder dominates can easily be obtained by looking at zero temperature. To obtain a reliable order of magnitude of the "disorder-induced melting" field $H_{M}$, it is necessary to know the precise $B(r)$ in the presence of disorder and not only its asymptotic forms. Fortunately such a calculation was performed in 16, 17]. Using formula (4.18) of Ref. [17] one gets

$$
B(r)=\frac{a^{2}}{\pi^{2}} \tilde{b}\left(r / R_{a}\right)
$$

For $r=R_{a}$ one has from [17] that $\tilde{b} \approx 1$ while for $r<R_{a}$ one is in the random manifold regime and one can approximate $B(r) \simeq \frac{a^{2}}{\pi^{2}}\left(r / R_{a}\right)^{1 / 3}$. From the solution of 17] we know that the above formula is quantitatively correct, and not only asymptotics. Using (5) one finds that

$$
a / R_{a}=\left(\pi c_{L}\right)^{6}
$$

Using $c_{L}=0.12$ gives $R_{a} \sim 350 a$. Thus the transition occurs well before the asymptotic regime. We will 
find that it does occur (e.g in BSCCO) indeed deep into the random manifold regime. One also notes that in simplified models without intermediate random manifold regime (where one directly go form a Larkin regime to the asymptotic regime) the above formula would give $a / R_{a}=\left(\pi c_{L}\right)^{2 /(4-d)}$. The transition then occurs for smaller values of $R_{a} / a$, in agreement with the results found in a special geometry [23.24.

Using (8) and the expression (4.12) of 17 for $R_{a}$ :

$$
R_{a}=\frac{2 a^{4} c_{66}^{3 / 2} c_{44}^{1 / 2}}{\pi^{3} \rho_{0}^{2} U_{p}^{2} d \xi^{2}}
$$

as well as $c_{66}=\epsilon_{0} /\left(4 a^{2}\right)$ and $c_{44} \approx c \epsilon_{0} /\left(\gamma^{2} a^{2}\right)$ (single vortex contribution) with $\epsilon_{0}=\left(\Phi_{0} / 4 \pi \lambda\right)^{2}$ and $c$ a numerical constant [32]. One gets:

$$
a^{3}=\frac{4 \pi^{3}}{\left(\pi c_{L}\right)^{6}} \frac{U_{p}^{2}}{\epsilon_{0}^{2}} d \xi^{2} \frac{\gamma}{\sqrt{c}}
$$

One thus obtains an expression for the transition field $H_{M}$ naturally expressed in terms of some characteristic fields of the system:

$$
H_{M}(T=0)=\frac{\left(\pi c_{L}\right)^{4}}{(16 \pi)^{1 / 3} \pi^{2}}\left(\frac{\epsilon_{0}}{U_{p}}\right)^{4 / 3} H_{c 2}^{2 / 3} H_{\text {cross }}^{1 / 3}
$$

where we have introduced the crossover field $H_{\text {cross }}=$ $\pi c \Phi_{0} /\left(\gamma^{2} d^{2}\right)$ with $c \sim \ln (\gamma d / \xi)$ 32 and $H_{c 2}=\Phi_{0} / \xi^{2}$.

As numerical estimate of the melting field $H_{M}$ for BiSCCO with $H_{\text {cross }} \sim 1 T, H_{c 2} \sim 100 T, U_{p} / \epsilon_{0}=0.4$, $c_{L}=.12$ gives $H_{M} \sim 400 G$ in good agreement with the observed experimental values [31]. The fact that this field is well below the decoupling field validates a posteriori the calculation ( note also that $B(r=0, z=d)$ is still small at the transition). The general shape of the phase diagram is in agreement with the one of Figure 1. Note that some non linear effects, such as screening of disorder by thermal fluctuations or by interactions at short scales may not be captured directly by the gaussian theory of 17]. They can be incorporated by a renormalization of the effective disorder $U_{p}(T)$. Such effects were computed in the flux liquid using RG in 7 and it was shown that the pinning length was renormalized upward (and thus the effective pinning strength downward) by a factor $\exp \left(\left(T / T_{d p}\right)^{3}\right)$ where $T_{d p} \sim\left(U_{p}^{2} d \xi^{2} c \epsilon_{0} / \gamma^{2}\right)^{1 / 3}$ is the single vortex depinning temperature [1]. It would be interesting to compute these effects in the solid as well. On general grounds that thermal fluctuations can only weaken the disorder, one expects an additional curvature upward of the Bragg glass instability line $H_{M}(T)$ when $T$ increases beyond $O\left(T_{d p}\right)$.

In Fig. 1 two main regions can be distinguished: if the temperature is high the stability line is nearly indistiguishable from the melting line of the pure system. This regime corresponds to the case where $R_{a}(T=0) \gg a$. In that case the translational order is only affected at distances huge compared to the lattice spacing, and the modification compared of a pure lattice is negligeable as far as the melting is concerned. This part of the stability line is therefore nearly identical to the melting of a pure lattice and one can expect the transition to be first order. The Bragg glass melts to a liquid phase, nearly insensitive to disorder.

If the field is increased one will shorten $R_{a}(T=0)$. The disorder itself is now able to make dislocation proliferate. In particular even at $T=0$ disorder destroys the Bragg glass. In this range of field and at low $T$ the transition line flattens as a function of temperature, since it is controlled mainly by the disorder. The phase into which the Bragg glass "melts" at low $T$ is relatively poorly understood. It is characterized by an absence of translational order and of Bragg peaks. There should still be some amount of pinning at low $T$, but whether such a phase is a true glass with diverging barriers, similar to the proposed vortex glass of Ref. [8], or simply a very viscous form of the liquid phase remains controversial. This phase could also retain hexatic order (hexatic glass) since, at least at a naive level, similar arguments for survival of hexatic order as for topological order in the Bragg glass can be given. If the phase is a true glass phase then it should melt thermally to the liquid, on the thick-dotted line of Fig. 1. Whether a true vortex glass phase exists in untwinned samples is an important still open and controversial question 37] which may need to be settled by high sensitivity 38] measurements. Since the low temperature phase is in any case much more continuously related to the liquid phase, one can expect now the transition to become second order. The Bragg glass therefore provides one natural explanation for a change of the order of the (thermal) melting transition, as well as for the existence of a field-induced transition.

One should also point out that at very low fields $\left(B \sim H_{c 1}\right)$ where screening is important $a>\lambda$, a similar (inverted) field driven transition should also occur when the field is lowered, from the Bragg glass to a pinned liquid (or another glass) as suggested by the decoration images of [13]. As shown in [7] the liquid becomes unstable for $B<B_{0} \exp \left(-\left(T / T_{d p}\right)^{3}\right)$, presumably to a glass or a pinned liquid.

The proposed field-induced transition between the Bragg glass and the putative vortex glass being just characterized by an injection of dislocations, it is not necessarily linked to a decoupling beween the layers. As a consequence one does not expect the critical current along $z$ to become zero at the field-induced transition, at least for low anisotropy system like YBCO. Of course it is always possible that in materials with high anisotropy like BSSCO dislocations prefer to appear first between the planes and the BG-VG and decoupling transition coincide. Let us however emphasize that it does not need to be so and that we also expect our transition to occur in purely isotropic systems. Another argument against the 
field-induced transtion being a simple thermal decoupling transition [32], is the fact that such a transition could not extend down to zero temperature. In any case measurements of the critical current perpendicular to the plane, in particular in YBCO, should help to separate between the two effects.

The suggestion that there may be two different glass phases could seem farfetched. There is a case however, mostly of theoretical interest at present, where it should happen as a direct consequence of our Bragg glass considerations. This is for $d$-dimensional vortex line systems with correlated disorder or equivalently in $d-1$ dimensional quantum bosons with disorder. It is reasonably well established theoretically, numerically and experimentally that a Bose glass phase exists for these systems in $d=3$ (i.e $d=2+1$ ( 2 space, 1 time dimension) for bosons). It is also believed that this phase lacks translational long range order in the plane perpendicular to the columns. Indeed since the vortices are localized along the columns, one can roughly view the properties in the perpendicular plane perpendicular, as those of a $d-1$ dimensional system with point-like disorder. For the $d=3$ vortex problem, dislocations are therefore expected to appear (as they presumably appear for $d=2$ systems with point like disorder). In higher dimension however, this need not be the case. For instance in $d=4$ for vortex systems ( $d=3+1$ for quantum particles) one is led, by similar arguments as in 17] to two distinct localized phases. For weak disorder no dislocation will appear, giving a Bose glass with topological order. This "BraggBose glass" phase is the equivalent for columnar defects of the Bragg glass one occuring for point-like disorder. For stronger disorder, dislocations will destroy the topological order perpendicular to the columns, giving back the "conventional Bose glass", i.e the continuation of its $d=2+1$ version. At the transition between these two different Bose glass phases, unbinding of dislocations loops (cylinders) should occur. An interesting point is that in the "conventional Bose glass" these dislocations loops will remain pinned to the colummnar defects, and it will thus be a true glass. This Bose glass phase would be in some sense analogous, in the case of point disorder, to the putative vortex glass. However, for pointlike disorder it is much less obvious that such a phase exists as a genuine thermodynamic phase. Let us note that this problem was studied analytically within an elastic theory in [35] using a variational method and in [36] using RG methods: the phase described there is thus the "Bragg-Bose glass". The difference between the two phases should occur only at scales larger than the distance between unpaired dislocations.

Several consequences of our theory could be further checked in experiments. First since the Bragg glass phase has translational order and the vortex glass has not, neutron experiments should observe a destruction of the Bragg peaks at the same location [34] than the transition observed by magnetic measurements. Such a feature seems to be consistent with the existing experimental data in BSSCO [28], but a more detailed experimental investigation would be needed to check this point in other materials as well. Another clear distinction between the two phases should be observed when a cycling in current similar to the one of [26] is performed. Such cycles taking the system above the critical $J_{c}$ and then back to zero, are expected to heal the lattice and to expel out of equilibrium dislocations. One can therfore expect good healing in the Bragg glass phase, as is indeed the case, since dislocations can only exist as out of equilibrium object. On the other hand the same cycle performed in the VG phase should make little difference on the neutron diffraction pattern since the equilibrium state already contains unpaired dislocations.

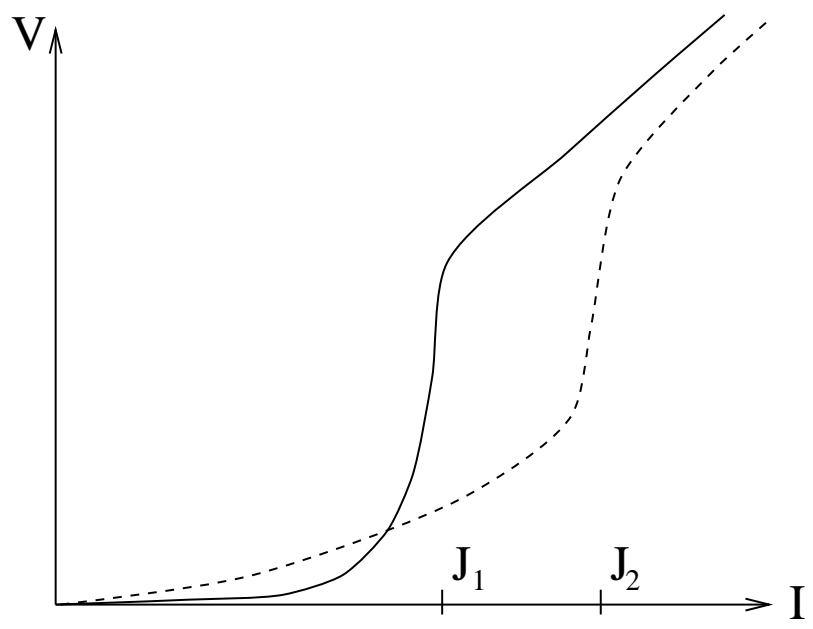

FIG. 2. $I-V$ characteristics in the Bragg glass phase and in the vortex glass (or pinned liquid) are shown schematically respectively as solid and dashed lines. One goes from small $\left(J_{1}\right)$ to larger $\left(J_{2}\right)$ critical currents, but rapidly divergent to weakly divergent (or finite) barriers, when increasing the field.

Finally one expects the barriers to vary very differently in the two glass phases. In the Bragg glass phase, elasticity is strong. Pinning can only be collective and one expects therefore weak barriers at short length scales. This implies a small critical current. On the other hand, since creep can only occur collectively, the barrier should grow very rapidly with decreasing current. A simple estimate gives $U(j) \sim(1 / j)^{\mu}$ with $\mu$ ranging between $\mu=0.7-0.8$ at intermediate currents to $\mu=0.5$ at small currents 117 while taking the dispersion of elastic moduli into account leads to higher values of $\mu$ in the intermediate regime [1]. On the other hand in the VG phase barriers should be significantly larger at short length scales since the nearly destroyed lattice has additional effective degrees of freedom, such as free dislocations, and can thus adapt more easily to the pinning 
potential. The critical current should therefore increase when approaching the field-induced transition. On the other hand in the VG phase the barriers should grow much more slowly with decreasing current since there is no need for collective motion, or even remain finite if the phase is simply a crossover from the liquid phase. Some estimates of the exponents for the gauge glass model gave very small exponents of the order of $\mu \sim 0.1-0.2$. One can therefore expect $I-V$ characteristics evolving with fields like the ones shown in Figure 2. Such a behavior is in good qualitative agreement of the observations of a second peak in relaxation measurements when the transition is passed. More refined transport, or relaxation measurement should help deciding on the behavior of the barriers.

It is important to note that the Lindemann criterion used here is not a detailed theory of the transition when dislocation proliferate, which is not yet available. It represents one possible mechanism of instability dominated by short length scales. It thus provides a reasonable upper bound for the instability field $H_{M}$ since the Bragg glass certainly cannot self-consistently survive if $R_{a}<a$. However, it cannot be excluded that because of the weakening of translational order at large distances in the Bragg glass compared to a real solid, unbound dislocations start to appear first at large length scales providing a different instability mechanism. In that case this additional phase (which may or may not be a true glass) would also melt through a first order transition with good short distance translational order properties.

In conclusion, we have examined in some details the implications, for the phase diagram of type II superconductors, of the existence of a glass phase with translational order: the Bragg glass. We have shown that it provides a natural interpretation of many of the features of the phase diagram of BSCCO and YBCO observed in experiments. Namely a crossover from a first order melting transition to a continuous transition when the field is increased, and the existence of a field induced transition. We interpret this last transition as the destruction of the Bragg glass phase by spontaneous injection of unbounded dislocations, into a topologically disordered glass phase or liquid. This transition, being disorder driven, should extend down to $T=0$.

* Laboratoire Associé au CNRS. email: giam@lps.upsud.fr.

** Laboratoire Propre du CNRS, associé á l'Ecole Normale Supérieure et à l'Université Paris-Sud. email: ledou@physique.ens.fr.

[1] G. Blatter et al., Rev. Mod. Phys. 66, 1125 (1994).

[2] D.R. Nelson, Phys. Rev. Lett. 691973 (1988).
[3] A. Houghton, R.A. Pelcovits and A. Sudbo, Phys. Rev. B 406763 (1989).

[4] P.L. Gammel, L. F. Schneemeyer, J.V. Waszcak and D. J. Bishop Phys. Rev. Lett. 611666 (1988).

[5] M. P. A. Fisher, Phys. Rev. Lett. 62, 1415 (1989).

[6] M. Feigelman, V. Geshkenbein, A. Larkin, and V. Vinokur, Phys. Rev. Lett. 63, 2303 (1989).

[7] D.R. Nelson and P. Le Doussal, Phys. Rev. B 4210113 (1990).

[8] D. S. Fisher and M. P. A. Fisher and D. A. Huse, Phys. Rev. B 43130 (1991).

[9] E. Chudnovsky, Phys. Rev. Lett. 653060 (1990).

[10] J. P. Bouchaud, M. Mézard, and J. Yedidia, Phys. Rev. Lett. 67, 3840 (1991) and Phys. Rev. B 46, 14686 (1992)

[11] M. Charalambous, J. Chaussy, and P. Lejay, Phys. Rev. B 45, 5091 (1992).

[12] H. Safar et al., Phys. Rev. Lett. 70, 3800 (1993).

[13] D. Grier et al. Phys. Rev. Lett. 662270 (1991).

[14] J. Villain and J. F. Fernandez, Z Phys. B 54, 139 (1984).

[15] T. Nattermann, Phys. Rev. Lett. 64, 2454 (1990).

[16] T. Giamarchi and P. Le Doussal, Phys. Rev. Lett. 72, 1530 (1994).

[17] T. Giamarchi and P. Le Doussal, Phys. Rev. B 52, 1242 (1995).

[18] In 17 we have computed the quantity $B(r)$ using the $\mathrm{RG}$ and a variational method. To then estimate the decay of translational order correlation function $C_{K}(r)=$ $\langle\exp (i K .(u(r)-u(0)))\rangle$ we have used that $C_{K}(r) \approx$ $\exp \left(-K^{2} B(r)\right) \sim 1 / r^{A_{d}}$, i.e a gaussian approximation. This is a reasonable lower bound for $C_{K}(r)$. It may give the exact asymptotic decay or it is also possible that atypical "return to the origin" events (i.e a singularity at $u=0$ of the scaled probability of $u$ ) could make this decay slower. A similar situation is discussed in 19 .

[19] P.P. Mitra, P. Le Doussal, Phys. Rev. B 4412035 (1991).

[20] One can in principle use a more realistic correlator (such as one with algebraic decay), but this should not change the general form of our results.

[21] M. J. P. Gingras and D. A. Huse Phys. Rev. B 53 (1996).

[22] S. Ryu, A. Kapitulnik and S. Doniach Phys. Rev Lett. 77 (1996). For an early discussion of the possible phase diagram see also A. Kapitulnik et al. SPIE proceedings 215712 (1994).

[23] J. Kierfeld, T. Nattermann, T. Hwa preprint condmat/9512101, to be published.

[24] D. Carpentier, P. Le Doussal and T. Giamarchi, Europhys. Lett. 35379 (1996) and to be published.

[25] W. Kwok et al. Physica B 197579 (1994)

[26] U. Yaron et al. Phys. Rev. Lett. 732748 (1994)

[27] T. Giamarchi and P. Le Doussal Phys. Rev. Lett. 753372 (1995).

[28] R. Cubbit et al. Nature 365407 (1993), Forgan et al. Preprint (1996).

[29] E. Zeldov et al. Nature 375373 (1995).

[30] B. Khaykovich et al. Phys. Rev. Lett. 762555 (1996).

[31] B. Khaykovich et al. preprint (1996).

[32] L. I. Glazman and A. E. Koshelev, Phys. Rev. B 43, 2835 (1991).

[33] A. I. Larkin and Y. N. Ovchinnikov, J. Low Temp. Phys 34, 409 (1979).

[34] How fast should one see the Bragg peaks disappear in ex- 
periments is unclear. The presence of disorder and temperature induced finite dislocation loops as a precursor near $H_{M}$ could make the neutron peaks disappear more gradually. We thank T. Nattermann for a discussion on this point.

[35] T. Giamarchi and P. Le Doussal Phys. Rev. B 5315206
(1996).

[36] L. Balents, Europhys. Lett. 24489 (1993).

[37] J. A. Fendrich et al. Phys. Rev. Lett. 741210 (1995).

[38] M. Charalambous et al., Phys. Rev. Lett. 752578 (1995).

[39] H. Safar et al. Phys. Rev. B 526211 (1995). 